\title{
Estudo das Propriedades Psicométricas do Inventário de Reconhecimento de Sinais de Alerta para Atos Suicidas
}

\author{
A Study on the Psychometric Properties of the Warning Signs Recognition for \\ Suicidal Acts Inventory
}

Marta Brás $^{1}$, Cláudia Carmo ${ }^{1}$ e Saul Neves de Jesus ${ }^{1}$

\begin{abstract}
Resumo
O reconhecimento de sinais de alerta pelos pares é uma estratégia fundamental para a prevenção do suicídio. Contudo, não existem instrumentos que avaliem o conhecimento dos adolescentes sobre esses sinais. Este estudo pretende desenvolver um Inventário de Reconhecimento de Sinais de Alerta para Atos Suicidas (IRSAS) e estudar as respetivas características psicométricas. A amostra integrou 512 adolescentes do ensino secundário (59\% do sexo feminino), com uma média de idades 16.78 anos. As análises fatoriais exploratória e confirmatória, juntamente com critérios teóricos, conduziram a uma solução trifatorial (23 itens) que avalia sinais de alerta comportamentais, verbais e de isolamento. O IRSAS apresentou características de fiabilidade e de validade bastante satisfatórias.
\end{abstract}

Palavras-chave: sinais de alerta, suicidio, adolescentes

\begin{abstract}
The recognition of warning signs by peers is a key strategy for suicide prevention. However, there are no tools to assess the knowledge of adolescents about these signs. This study aims to develop a Warning Signs Recognition for Suicidal Acts Inventory (WSSAI) and study the respective psychometric characteristics. The sample comprised 512 high-school adolescents (59\% female) with a mean age16.78 years. Exploratory and confirmatory factor analyzes, along with theoretical criteria, led to a three-factor solution (23 items) that assesses behavioral, verbal and isolation warning signs. The WSSAI presented quite satisfactory reliability and validity characteristics.
\end{abstract}

Keywords: warning signs, suicide, adolescents 


\section{Introdução}

Atualmente morrem aproximadamente 1.1 milhão de pessoas por suicídio num ano, sendo esta a segunda causa de morte na faixa etária dos 15 aos 29 anos (OMS, 2015). Em Portugal, o suicídio também representa um preocupante problema de saúde pública e os jovens estão sinalizados como um grupo de risco, no recente Plano Nacional de Prevenção do Suicídio (DGS, 2013).

O reconhecimento dos sinais de alerta é um dos princípios básicos para a prevenção dos atos suicidas, sendo uma componente transversal aos vários níveis de prevenção: primária, secundária e terciária. No âmbito da prevenção primária, em específico, ter a capacidade de identificar os sinais de alerta, pode ser determinante na interrupção precoce do percurso suicida, antes da ocorrência do primeiro ato.

Deste modo, pressupõe-se que a divulgação dos sinais de alerta em campanhas de prevenção potencia a sua deteção precoce, assim como o encaminhamento de indivíduos vulneráveis para intervenção clínica e, consequentemente, a redução do número de mortes por suicídio (Rudd, Berman, et al., 2006; Shaffer \& Gould, 2000).

No entanto, o estudo dos sinais de alerta para os atos suicidas tem encontrado algumas dificuldades conceptuais e metodológicas. Começando pela definição, o constructo sinais de alerta, também designado por sinais de risco ou sinais de perigo, para os atos suicidas, permaneceu, durante largos anos, indefinido e pouco estudado na literatura científica (Rudd, Mandrusiak, et al., 2006). Apenas recentemente foram desenvolvidos alguns esforços para circunscrever este constructo e distingui-lo de outros próximos, com os quais é muitas vezes confundido, como é o caso dos fatores de risco (e.g., Rudd, Berman, et al., 2006).

Os sinais de alerta para o suicídio podem ser definidos como "manifestações comportamentais da precipitação de condições num indivíduo particular: elas são diretamente observáveis, refletem o atual estado do indivíduo e indicam a presença de uma crise suicida" (Van Orden et al., 2006, p. 273). Os sinais de alerta sugerem, assim, a possibilidade de ocorrência de um ato suicida num futuro muito próximo - que pode variar de horas a alguns dias - e, por isso, exigem uma intervenção imediata e eficaz (Hawton, 2010; Rudd, Berman, et al., 2006). Estes sinais caracterizam-se também por serem dinâmicos, episódicos e variáveis (e.g., ideação suicida frequente; plano suicida), o que significa que podem desaparecer com alguma facilidade quando a crise suicida é superada. O mesmo não se sucede com os fatores de risco que são mais estáveis, persistentes, com uma duração mais prolongada no tempo e com menos probabilidade de mudança (e.g., o diagnóstico de uma doença psiquiátrica; características de personalidade; família disfuncional) (Rudd, Berman, et al., 2006). Além disso, os sinais de alerta têm um carácter tendencialmente mais subjetivo (e.g., ameaças de suicídio) e os fatores de risco são mais objetivos (e.g., história de tentativas de suicídio) (Rudd, Berman, et al., 2006). Assim, um indivíduo pode apresentar vários fatores de risco que o tornam mais vulnerável para um ato suicida no futuro, mas não significa que está em perigo iminente de cometer este ato. Normalmente, o processo suicidário desenrola-se após a ocorrência de um acontecimento precipitante (e.g., rutura amorosa) num indivíduo que já apresenta fatores de risco. Neste contexto, para além dos fatores de risco, o indivíduo começa a manifestar sinais de alerta que indicam um sofrimento psicológico significativo no presente $\mathrm{e}$, consequentemente, uma maior probabilidade de ocorrência de um ato suicida num futuro próximo.

Se estas características concetuais dos sinais de alerta são consensuais, o mesmo não acontece com a identificação e importância comparativa dos vários sinais de alerta, que podem significar a iminência de um ato suicida.

A identificação dos sinais de alerta específicos para os atos suicidas tem suscitado alguma controvérsia devido à própria complexidade do fenómeno suicidário. Alguns indivíduos apresentam vários sinais de alerta, mas não cometem atos suicidas; outros mostram poucos ou nenhuns sinais de alerta e realizam atos suicidas, $o$ que significa que há sinais de alerta comuns em indivíduos suicidas e não-suicidas. Além disso, os sinais de alerta podem não ser os mesmos em diferentes indivíduos e assumirem diferentes 
graus de importância (Joiner, 2010; Miller, 2011; Shaffer \& Gould, 2000), uma vez que a personalidade e a resiliência dos adolescentes condicionam os comportamentos de risco em geral (Carvalho \& Novo, 2014) e o risco suicida em particular (Arenas-Landgrave, Maqueo, \& Forns, 2012).

Perante as dificuldades concetuais e a ausência de um documento oficial com o conjunto dos sinais de alerta, um grupo de trabalho sob os auspícios da Associação Americana de Suicidologia (AAS), em 2003, elaborou uma lista desses potenciais sinais organizada de forma hierárquica e dividida em duas partes. $\mathrm{Na}$ primeira, foram apresentados os sinais que carecem de intervenção muito urgente, nomeadamente ameaças suicidas, planos suicidas e expressão verbal ou escrita de pensamentos suicidas ou sobre a morte. $\mathrm{Na}$ segunda, foram expostos os sinais que carecem de avaliação por parte de profissionais especializados, mas não com carácter de máxima urgência. Desta segunda lista constam os seguintes sinais: (a) desesperança; (b) raiva e desejo de vingança; (c) envolvimento em atividades de risco, aparentemente de forma impulsiva; (d) sentimento de "estar preso", sem qualquer saída; (e) aumento do uso de álcool e drogas; (f) isolamento dos amigos, família ou sociedade; (g) ansiedade, agitação, dificuldades para dormir ou dormir excessivamente; (h) mudanças drásticas do humor e (i) ausência de razões para viver, perceção de que a vida não tem sentido, nem objetivos (AAS, 2013).

A AAS criou ainda a mnemónica IS PATH WARM? que corresponde aos seguintes sinais de alerta: (a) ideação suicida (I-Ideation); (b) abuso de substâncias (S-Substance Abuse); (c) sentimento de inutilidade (P-Purposelessness); (d) ansiedade (A-Anxiety); (e) sentimento de que não existem alternativas (T-Trapped); (f) desesperança (H-Hopelessness); (g) isolamento (WWithdrawal); (h) raiva (A-Anger); (i) impulsividade (R-Recklessness) e (j) mudanças de humor (M-Mood Changes). Esta mnemónica está disponível no sítio web da AAS (2013), sendo largamente difundida em newsletters (e.g., Juhnke, Granello, Lebrón-Striker, \& LebrónStriker, 2007) e em artigos científicos (e.g.,
Bryan, Steiner-Pappalardo, \& Rudd, 2009; Rudd, Berman, et al., 2006).

Os sinais de alerta têm sido categorizados também de outras formas: (a) sinais verbais diretos; (b) sinais verbais indiretos; (c) sinais comportamentais diretos e (d) sinais comportamentais indiretos.

Os sinais verbais diretos são expressões diretas dos pensamentos suicidas, como por exemplo "eu quero morrer". Os sinais verbais indiretos correspondem a expressões que indiciam o desejo de morrer, como sejam "apetece-me desaparecer". Os sinais comportamentais diretos são ações relacionadas com a preparação dos atos suicidas, de que pode ser exemplo a compra de produtos tóxicos. Os sinais comportamentais indiretos correspondem a indícios de que a pessoa pode estar a preparar o suicídio, como por exemplo o ato de se desfazer dos seus objetos pessoais significativos (Inoue et al., 2007).

Com base na categorização em estudo, numa amostra com 2948 indivíduos que cometeram atos suicidas, em que $40.4 \%$ dos casos demonstraram sinais de alerta, verificou-se que $32.5 \%$ eram sinais verbais diretos, $27.8 \%$ sinais verbais indiretos, $6.5 \%$ sinais comportamentais diretos e $33.2 \%$ sinais comportamentais indiretos (Inoue et al., 2007).

Os sinais de alerta enunciados na investigação anterior são comuns aos potenciais suicidas de várias faixas etárias. $\mathrm{O}$ facto de não existir uma lista de sinais de alerta validada para a população jovem que seja distinta das listas de outras faixas etárias, é outra das lacunas apontadas no âmbito da Suicidologia na adolescência (Miller, 2011). Existem porém alguns autores que, nas últimas décadas, se têm dedicado especificamente ao estudo dos atos suicidas e dos sinais de alerta na adolescência, dadas as particularidades dos indivíduos nesta fase de desenvolvimento.

Os adolescentes podem falar abertamente sobre a morte e o morrer (Berman, Jobes, \& Silverman, 2006), mas o mais comum é transmitirem os seus pensamentos suicidas apenas por pistas indiretas, sem os verbalizarem diretamente (Capuzzi, 2009). Entre essas pistas podem enunciar-se desenhos sobre a morte, a falta de cuidado com a segurança pessoal, envolvimento em comportamentos de risco pouco 
habituais, como conduzir a alta velocidade ou experimentar drogas, álcool e/ou medicamentos em excesso (Berman et al., 2006; Capuzzi, 2009). Também ao nível dos padrões sociais, um adolescente cooperante, compreensivo e socialmente participativo pode tornar-se indiferente aos outros, egoísta, desrespeitador das regras e isolar-se das atividades em que habitualmente participava. A diminuição repentina do desempenho e interesse escolar é outra área que pode indiciar a presença de pensamentos suicidas, pois os adolescentes deixam de ter concentração suficiente para se envolverem e investirem nas tarefas escolares (Capuzzi, 2009).

Entre os sinais de alerta nos adolescentes, podem, ainda, referir-se dificuldades de concentração, alteração dos padrões de sono e de alimentação, tentativas para colocar os assuntos e objetos pessoais em ordem, preocupações com a morte ou com temas violentos e melhoria repentina após um período depressivo (Capuzzi, 2009).

Em termos de linguagem verbal, alguns adolescentes podem expressar-se aberta e diretamente através de frases do tipo "eu vou-me matar" ou "eu estou a pensar terminar com a minha vida" ou, de forma menos direta, "eu voume embora de casa", "ela vai arrepender-se da forma como me tem tratado", "um dia vão aperceber-se que as coisas que eu dizia eram sérias" (Capuzzi, 2009).

Como se referiu anteriormente, os sinais de alerta não são os mesmos para todos os indivíduos e nem todos manifestam esses sinais. Contudo, a maioria dos adolescentes que comete atos suicidas expressa sinais de alerta (King, 1999), embora esses sinais sejam geralmente ignorados ou malinterpretados pelos amigos e pela família (Barnes, Ikeda, \& Kresnow, 2001; Berman et al., 2006). Como grande parte dos indivíduos $(75 \%)$ que se suicidam não estão em contacto com os serviços de saúde (Owens, Booth, Briscoe, Lawrence, \& Lloyd, 2003) manifestando apenas esses sinais entre os familiares e amigos, torna-se essencial prestar informação sobre os mesmos aos entes mais próximos (Hjelmeland \& Knizek, 2004). A informação sobre os sinais de alerta é igualmente importante para as pessoas vulneráveis, de modo a que possam solicitar ajuda precocemente (Aseltine \& DeMartino, 2004; Aseltine, James, Schilling, \& Glanovsky, 2007; Capuzzi, 2009).

Face ao exposto, torna-se essencial desenvolver programas de consciencialização sobre a prevenção do suicídio que incluam os sinais de alerta. $\mathrm{O}$ aumento do conhecimento sobre os sinais de alerta, enquanto estratégia de prevenção primária (Gould et al., 2005), tem como objetivo último tornar a deteção do processo suicida mais precoce (Gould, Greenberg, Velting, \& Shaffer, 2003; Miller, 2011; Shaffer \& Gould, 2000), de modo a facilitar uma intervenção mais célere e eficaz (Berman et al., 2006; Miller, 2011; Rudd, Berman, et al., 2006).

No entanto, os instrumentos para avaliar o reconhecimento de sinais de alerta com características psicométricas satisfatórias são escassos, sobretudo em adolescentes. Podemos referir a lista de sinais de alerta publicada pela AAS, já mencionada anteriormente, que é um instrumento de sensibilização, mas não é um instrumento de medida cujas características psicométricas tenham sido estudadas, ou o Awareness of Suicide Warning Signs Questionnaire (Tsai, Lin, Chang, Chang, \& Chou, 2010), que se refere a sinais de alerta da população adulta e tem como destinatários também adultos. Em língua portuguesa também não se conhece a existência de um instrumento que permita medir o reconhecimento de sinais de alerta para os atos suicidas em adolescentes, avaliar a aquisição de conhecimentos, após uma ação de prevenção, e cujas propriedades psicométricas sejam adequadas, o que motivou o desenvolvimento de um inventário com estas características.

O presente estudo teve como principal objetivo desenvolver um Inventário de Reconhecimento de Sinais de Alerta para Atos Suicidas (IRSAS) nos adolescentes e estudar as respetivas características psicométricas. Neste sentido, foram delineados os seguintes objetivos específicos: (a) inventariar os sinais de alerta para o suicídio em adolescentes; (b) averiguar a validade de conteúdo do inventário; (c) analisar as características descritivas e a validade interna dos itens; (d) estudar as características psicométricas, nomeadamente a estrutura interna, a fiabilidade 
(consistência interna e estabilidade temporal), a validade convergente e a validade discriminante, bem como a validade por referência a critério e (e) explorar as diferenças no inventário em função do sexo dos participantes.

\section{Método}

\section{Estratégias para a construção e desenvolvimento do Inventário de Reconhecimento de Sinais de Alerta para Atos Suicidas (IRSAS)}

A conceção do presente inventário obedeceu a um conjunto de procedimentos estabelecidos na literatura (Almeida \& Freire, 2008; Gutierrez \& Osman, 2008; Hill \& Hill, 2008; Moreira, 2004; Ribeiro, 1999), quer para a elaboração dos itens, instruções e escala de medida, quer para o estudo das suas características psicométricas.

Começou-se por definir o constructo-alvo e o objetivo do inventário, bem como a população a que se destina e o contexto de aplicação.

O constructo-alvo consiste nos sinais de alerta para os atos suicidas, sendo este definido como manifestações diretamente observáveis que sugerem o risco do indivíduo cometer um ato suicida num futuro próximo (Van Orden et al., 2006).

Este inventário tem como principal objetivo avaliar em que medida os adolescentes concordam ou discordam que cada um dos comportamentos ou verbalizações apresentados pode representar um sinal de alerta para o ato suicida, de um adolescente.

No que respeita aos destinatários, são os adolescentes, sendo o contexto de aplicação, por conveniência, os estabelecimentos de ensino secundário.

Tendo em consideração a diversidade de sinais de alerta e as especificidades inerentes a este constructo, concebeu-se o inventário como uma medida unidimensional, tendo-se prosseguido com a elaboração dos itens, baseados na literatura sobre o tema. O conteúdo dos itens resultou da compilação de sinais de alerta apresentados por autores da área da Suicidologia na adolescência (e.g., Berman et al., 2006; Capuzzi, 2009; Miller, 2011; Rudd, Berman, et al., 2006; Shaffer \& Gould, 2000). Procedeu-se, posteriormente, à redação destes sinais com uma linguagem apropriada para adolescentes.

$\mathrm{O}$ número de itens inicial (37) foi aproximadamente o dobro do número (20) que alguns autores recomendam preservar na versão final dos inventários, após as análises estatísticas (Almeida \& Freire, 2008; Ribeiro, 1999). Assim, para que, numa fase seguinte, se pudesse selecionar os itens que apresentam caraterísticas psicométricas mais adequadas e excluir os restantes, alguns foram concebidos como sinónimos do ponto de vista conceptual, embora com redações diferentes. Todos os itens corresponderam a sinais de alerta para o suicídio formulados pela positiva.

As instruções de preenchimento consistiram em solicitar o grau de concordância com cada uma dessas afirmações: "[I]ndica em que medida concordas ou discordas que os seguintes sinais podem indicar que um(a) jovem está desesperado(a) ao ponto de pensar pôr termo à própria vida. Assinala com uma cruz $[\times]$, de acordo com a seguinte escala".

Relativamente à escala de resposta, optou-se por um formato tipo Likert de cinco pontos, desde 1 (Discordo completamente) a 5 (Concordo completamente).

A versão inicial do IRSAS foi submetida a um estudo piloto, de forma a avaliar a sua validade de conteúdo, com base nos procedimentos e critérios apresentados por Gutierrez e Osman (2008). O IRSAS foi analisado inicialmente por um conjunto de cinco juízes especialistas em Psicologia Clínica, com prática de investigação científica. Numa primeira fase, este painel de especialistas analisou os itens com base numa escala tipo Likert de cinco pontos, relativamente aos seguintes critérios: relevância, de 1 (Nada relevante) a 5 (Extremamente relevante); representatividade, de 1 (Nada representativo) a 5 (Extremamente representativo); especificidade, de 1 (Nada específico) a 5 (Extremamente específico); clareza, de 1 (Nada claro) a 5 (Extremamente claro); linguagem, de 1 (Nada adequada) a 5 (Extremamente adequada) e extensão, de 1 (Extremamente extenso) a 5 (Nada extenso). Numa fase seguinte, pediu-se que dessem um parecer sobre a clareza das instruções, a adequabilidade da escala de medida, a extensão 
global do inventário, a potencial utilidade do mesmo no âmbito da prevenção primária, a existência de eventuais sinais não incluídos pelos autores, assim como outras sugestões que considerassem pertinentes.

Após as reformulações decorrentes do parecer dos juízes, o IRSAS foi aplicado a um conjunto de seis adolescentes através da técnica "reflexão falada". A versão final do inventário ficou assim composta pelos 37 itens.

\section{Participantes}

A amostra do presente estudo integrou 512 jovens do ensino secundário, 210 do sexo masculino (41\%) e 302 do sexo feminino (59\%), com idades compreendidas entre os 15 e os 19 anos $(M=16.78 ; D P=1.17)$. A maioria desses jovens $(66.2 \%)$ residia com ambos os pais, 141 com o pai ou com a mãe, 22 com outros familiares e 10 com outras pessoas. O rendimento escolar médio da maioria $(55.5 \%)$ era considerado suficiente (10 a 13 valores), seguindo-se a classificação de bom (36.5\%), Muito Bom (5.7\%) e Insuficiente (2.3\%).

A amostra total de 512 jovens foi subdividida aleatoriamente em duas subamostras com $\mathrm{o}$ mesmo número de sujeitos, sendo que uma subamostra foi utilizada para realizar a análise fatorial exploratória e a outra subamostra para a análise fatorial confirmatória.

A primeira subamostra foi composta por 256 jovens, 149 do sexo feminino e 107 do sexo masculino, com uma idade média de 16.89 $(D P=1.123)$ anos. A segunda subamostra incluiu também 256 jovens, 153 do sexo feminino e 103 do sexo masculino, com uma média de idades de $16.64(D P=1.173)$ anos.

Num segundo momento de aplicação, três a quatro semanas depois, destinado à avaliação da fiabilidade pelo método teste-reteste, participaram os 300 jovens $(58.6 \%$ da amostra total) que preencheram novamente o IRSAS. As características sociodemográficas da subamostra $(N=300)$ e da amostra total $(N=512)$ foram comparadas. Os resultados não registaram diferenças estatisticamente significativas nem na idade $\left(t_{(437.882)}=-1.524, p>.128\right)$, nem no sexo $\left(\chi_{(1)}^{2}=.309, p>.578\right)$.

\section{Procedimento de Recolha de Dados}

Foi realizado, primeiro, um pedido de autorização à Direção-Geral de Inovação e de Desenvolvimento Curricular (DGIDC), do Ministério da Educação português, de seguida, aos estabelecimentos de ensino e aos encarregados de educação e, posteriormente, pediu-se o consentimento informado dos jovens. A aplicação do protocolo decorreu em contexto de sala-deaula, sempre na presença do primeiro autor deste estudo.

De forma a analisar a estabilidade temporal, a subamostra de 300 participantes anteriormente referida preencheu o IRSAS, uma segunda vez, num intervalo de tempo que oscilou entre as três e as quatro semanas, seguindo os mesmos procedimentos da primeira aplicação.

\section{Procedimento de Análise Estatística}

A maioria das análises estatísticas foi realizada com recurso ao programa de tratamento de dados estatísticos Statistical Package for the Social Sciences (SPPS) (versão 21.0) para Windows. Utilizou-se ainda o programa Microsoft Office Excel 2010 para calcular as estimativas das magnitudes do efeito obtidas através da fórmula para o $d$ de Cohen (1988) e o software Analysis of Moment Structures (AMOS) (versão 21.0) para realizar a análise fatorial confirmatória do IRSAS.

O estudo das propriedades psicométricas dos itens do IRSAS começou pela análise das medidas de tendência central (média), medidas de dispersão (desvios-padrão e amplitude), medidas de distribuição (assimetria e curtose) e medidas de precisão para avaliar a validade interna (sensibilidade e o poder discriminativo dos itens).

A análise fatorial exploratória foi desenvolvida de acordo com os seguintes critérios: selecionou-se o método das componentes principais; optou-se por rotação oblíqua (por não implicar que os fatores sejam independentes); retiveram-se os fatores com valor próprio superior à unidade e retiveram-se os itens com "pesos fatoriais" $\geq .40$ no fator primário.

A análise fatorial confirmatória foi efetuada para avaliar a qualidade do ajustamento aos dados. De acordo com os parâmetros estabelecidos na literatura (Kline, 2011; Marôco, 2010), considerou-se que o ajustamento de um modelo 
aos dados é adequado para valores do quociente do qui-quadrado pelos respetivos graus de liberdade $\left(\chi^{2} / \mathrm{gl}\right)$ inferior a 2-3; para valores de Comparative fit index (CFI), Goodness-of-fit index (GFI) e Tucker-Lewis index (TLI) iguais ou superiores a .9; para valores de Root mean square error of approximation (RMSEA) inferiores a .05 ou, para casos menos exigentes, inferiores a .08 ; para valores de Standardized root mean square residual (SRMSR) inferiores a .1 e para valores de Parsimonious normed fit index (PNFI) entre .5 e .9; ao compararem-se dois modelos, aquele com menores Expected cross-validation index (ECVI) e Akaike Information Criterion (ACAIC) corresponde ao mais estável na população.

Foi ainda estudada a fiabilidade do IRSAS através da análise da consistência interna (alfa de Cronbach) e da estabilidade temporal (método teste-reteste), bem como a validade convergente (índice da variância extraída média, VEM), a validade discriminante (variância partilhada, $r^{2}$ ) e a validade por referência a critério (coeficiente de correlação de Pearson entre o IRSAS e as medidas de resultado).

Em relação à análise da validade por referência a critério, uma vez que, de acordo com a revisão da literatura, não se conhecem medidas com características psicométricas adequadas para avaliar o reconhecimento dos sinais de alerta para os atos suicidas na população jovem, foram utilizadas três medidas de resultado: (a) perceção de sucesso da prevenção do suicídio; (b) perceção de autoeficácia na prevenção do suicídio dos pares e (c) perceção de sucesso na prevenção do suicídio do próprio, avaliadas numa escala tipo Likert. Assim sendo, a predisposição para a prevenção, na sua globalidade, foi avaliada através de três itens que correspondiam a afirmações. Solicitou-se aos jovens que assinalassem, com base numa escala tipo Likert de

1 (Discordo totalmente) a 5 (Concordo totalmente), em que medida concordavam com cada uma das seguintes afirmações: (1) É possível evitar que alguém termine com a própria vida; (2) Se um colega quisesse terminar com a própria vida, eu seria capaz de ajudá-lo a mudar de intenção e (3) Se eu pensasse em terminar com a minha vida, seria capaz de procurar ajuda (profissional ou outra) para desistir dessa ideia.
Estes três itens em conjunto apresentaram um alfa de Cronbach de .73, indicador da sua consistência interna.

Perspetivava-se, com base na literatura, que a uma maior concordância entre os sinais de alerta para os atos suicidas corresponderia uma maior predisposição para a prevenção do suicídio (Berman et al., 2006; Capuzzi, 2009; Kalafat \& Elias, 1994; Miller, 2011; Tsai et al., 2010; Waldvogel, Rueter, \& Oberg, 2008; Walker, Ashby, Hoskins, \& Greene, 2009).

Por último, foram exploradas diferenças entre grupos (sexo) com recurso ao test $t$ de Student para amostras independentes.

\section{Resultados}

O estudo do IRSAS começou pela análise das estatísticas descritivas dos itens (Quadro 1).

Os valores médios oscilaram entre o mínimo de 2.89 ( $D P=1.057)$, no item 29 "Dificuldade em dormir ou dormir demais, quando isso não é habitual" e o valor máximo de $3.82(D P=.853)$ no item 25 "Mostrar desinteresse pela vida". Estes resultados mostram que globalmente as médias foram superiores ao ponto intermédio da escala de resposta. Os desvios-padrão foram relativamente próximos da unidade, o que sugere uma dispersão adequada dos resultados, ou seja, estes encontramse relativamente próximos da média e não muito dispersos pela escala de resposta. Em relação à amplitude, em todos os itens foram registados os valores extremos da escala de resposta.

No que respeita às medidas de distribuição, foram obtidos valores de assimetria (entre -.77 e .02 ) e de curtose (entre -.75 e .35 ), que asseguram a existência de sensibilidade (valores absolutos inferiores a 1) (Marôco, 2011). Observou-se, ainda, que todos os valores de assimetria foram negativos, refletindo a preferência pelos valores mais elevados da escala de resposta de tipo Likert, como demonstraram também as médias dos itens. Os coeficientes de curtose, por sua vez, apresentaram sinais positivos e negativos, mas foram todos inferiores à unidade, indicando uma distribuição mesocúrtica.

Os coeficientes de correlação item $\mathrm{x}$ total corrigido registaram o valor mínimo de .41 , sugerindo que a validade interna dos itens é 
Quadro 1. Descrição Estatística dos Itens do IRSAS

\begin{tabular}{|c|c|c|c|c|c|c|}
\hline Itens & $M$ & $D P$ & $A$ & $C$ & ritc & $\begin{array}{c}\text { alfa s/ } \\
\text { item }\end{array}$ \\
\hline 1. Diminuição repentina do rendimento e interesse escolar habitual. & 2.97 & 1.049 & -.29 & -.75 & .41 & .96 \\
\hline 2. Isolamento social, afastamento das pessoas com quem convivia habitualmente. & 3.56 & .896 & -.59 & -.11 & .65 & .95 \\
\hline $\begin{array}{l}\text { 3. Desinteresse inesperado por atividades de que gostava muito (ex. jantares de } \\
\text { grupo,...) }\end{array}$ & 3.22 & 1.010 & -.35 & -.45 & .49 & .96 \\
\hline 4. Desabafos de que a sua vida não faz sentido. & 3.57 & .979 & -.77 & .18 & .58 & .96 \\
\hline $\begin{array}{l}\text { 5. Mensagens nas redes sociais (facebook, } m s n \text { ) ou noutros sítios web revelando } \\
\text { desespero. }\end{array}$ & 3.26 & 1.061 & -.54 & -.40 & .49 & .96 \\
\hline 6. Desvalorização dos projetos para o futuro. & 3.36 & .911 & -.34 & -.32 & .59 & .95 \\
\hline 7. Relacionamento problemático com a família e/ou o grupo de amigos. & 3.58 & .934 & -.48 & -.24 & .60 & .95 \\
\hline 8. Manifestações físicas de irritabilidade fora do que é comum. & 3.26 & .980 & -.16 & -.46 & .60 & .95 \\
\hline 9. Frases do tipo: "Estou farto(a) desta vida". & 3.27 & 1.099 & -.31 & -.71 & .59 & .96 \\
\hline 10. Aumento repentino do consumo de substâncias de risco (álcool, drogas,...). & 3.48 & 1.017 & -.34 & -.57 & .56 & .96 \\
\hline 11. Transmitir sentimentos de desapontamento face ao mundo e aos outros. & 3.46 & .866 & -.45 & -.07 & .61 & .95 \\
\hline 12. Verbalização de ideias associadas à morte. & 3.42 & .956 & -.49 & -.16 & .50 & .96 \\
\hline 13. Conversas que revelem desinteresse pelo "valor" da vida. & 3.65 & .832 & -.67 & .35 & .62 & .95 \\
\hline 14. Falar frequentemente sobre atos suicidas. & 3.42 & .996 & -.27 & -.58 & .56 & .96 \\
\hline 15. Expressões de pessimismo e desesperança em relação ao futuro. & 3.41 & .896 & -.44 & -.37 & .61 & .95 \\
\hline 16. Resultados escolares bastante inferiores ao esperado. & 2.93 & .995 & -.05 & -.58 & .56 & .96 \\
\hline 17. Afastamento repentino do grupo de amigos. & 3.35 & .957 & -.33 & -.55 & .66 & .95 \\
\hline 18. Expressões de insatisfação com a vida na sua globalidade. & 3.45 & .889 & -.39 & -.14 & .66 & .95 \\
\hline 19. Comunicação cada vez menos aberta com as pessoas próximas. & 3.43 & .903 & -.39 & -.17 & .65 & .95 \\
\hline $\begin{array}{l}\text { 20. Aborrecimento excessivo e constante com assuntos que antes considerava } \\
\text { agradáveis. }\end{array}$ & 3.33 & .901 & -.32 & -.26 & .63 & .95 \\
\hline $\begin{array}{l}\text { 21. Comportamentos de risco pouco habituais para essa pessoa (ex. beber } \\
\text { excessivamente, conduzir a alta velocidade). }\end{array}$ & 3.46 & .938 & -.46 & -.17 & .64 & .95 \\
\hline 22. Aumento do interesse e das conversas sobre o tema do suicídio. & 3.25 & .975 & -.33 & -.28 & .49 & .96 \\
\hline 23. Relacionamento conflituoso com as pessoas próximas. & 3.34 & .903 & -.35 & -.25 & .62 & .95 \\
\hline 24. Redução drástica da paciência para as pessoas. & 3.20 & .948 & -.16 & -.45 & .64 & .95 \\
\hline 25. Mostrar desinteresse pela vida. & 3.82 & .853 & -.62 & .02 & .62 & .95 \\
\hline 26. Ameaçar que vai cometer atos suicidas. & 3.58 & 1.011 & -.49 & -.31 & .49 & .96 \\
\hline 27. Falta de paciência repentina para qualquer assunto. & 3.16 & .880 & -.23 & -.13 & .57 & .96 \\
\hline 28. Dizer que não tem razões para viver. & 3.68 & .941 & -.77 & .43 & .59 & .95 \\
\hline 29. Dificuldade em dormir ou dormir demais, quando isso não é habitual. & 2.89 & 1.057 & -.11 & -.61 & .51 & .96 \\
\hline 30. Isolamento da família. & 3.57 & .942 & -.65 & .06 & .67 & .95 \\
\hline 31. Isolamento da sociedade. & 3.61 & .931 & -.73 & .34 & .70 & .95 \\
\hline 32. Dizer que se sente sem saída, sem alternativas para resolver os problemas. & 3.59 & .983 & -.58 & -.10 & .66 & .95 \\
\hline 33. Manifestações de raiva descontrolada. & 3.29 & .933 & -.30 & -.54 & .68 & .95 \\
\hline 34. Agir de forma imprudente, insensata, o que não é comum em si. & 3.31 & .938 & -.45 & -.15 & .66 & .95 \\
\hline $\begin{array}{l}\text { 35. Envolver-se em atividades de alto risco, quando essas atividades não eram } \\
\text { praticadas com regularidade. }\end{array}$ & 3.44 & .943 & -.33 & -.33 & .64 & .95 \\
\hline 36. Mostrar drásticas mudanças de humor. & 3.12 & .919 & -.02 & -.39 & .62 & .95 \\
\hline 37. Mostrar ansiedade ou agitação descontrolada, fora do comum. & 3.23 & .911 & -.29 & -.36 & .66 & .95 \\
\hline
\end{tabular}


adequada, bem como os valores do alfa de Cronbach global que foram sempre muito satisfatórios, mesmo que se eliminasse qualquer um dos itens.

Conhecidas as estatísticas descritivas, prosseguiu-se com a identificação do número de fatores a reter. A análise do scree plot sugeriu uma solução de 2 ou 3 fatores e a análise paralela com base numa Simulação de Monte Carlo, considerando 100 repetições, indicou 3 fatores, o que levou ao estudo de duas soluções fatoriais, uma com dois fatores e outra com três fatores.
Repetiram-se os procedimentos da análise fatorial exploratória até se reunirem os critérios de qualidade estabelecidos, pelo que foi necessário eliminar progressivamente alguns itens com comunalidades inferiores a .35 ou com pesos de saturação superiores a .4 nos fatores secundários. Após quatro fatorializações, estudaram-se as seguintes soluções fatoriais: $2 \mathrm{~F}$ (19 itens) e $3 \mathrm{~F}$ ( 23 itens), sendo que a solução de 3 fatores com 23 itens foi a que reuniu resultados mais satisfatórios (e.g., maior percentagem de variância total explicada, itens com "pesos fatoriais" mais

Quadro 2. Análise Fatorial Exploratória do IRSAS: Método das Componentes Principais, com Rotação Oblíqua, 3 fatores (23 itens)

\begin{tabular}{|c|c|c|c|c|}
\hline Itens & $\mathrm{F} 1$ & F2 & F3 & $h^{2}$ \\
\hline 36. Mostrar drásticas mudanças de humor. & .829 & .000 & .073 & .623 \\
\hline 24. Redução drástica da paciência para as pessoas. & .793 & -.030 & -.001 & .610 \\
\hline 27. Falta de paciência repentina para qualquer assunto. & .790 & -.025 & .063 & .555 \\
\hline 37. Mostrar ansiedade ou agitação descontrolada, fora do comum. & .706 & .000 & -.123 & .614 \\
\hline 34. Agir de forma imprudente, insensata, o que não é comum em si. & .682 & .179 & .027 & .578 \\
\hline 29. Dificuldade em dormir ou dormir demais, quando isso não é habitual. & .637 & -.080 & -.050 & .404 \\
\hline 8. Manifestações físicas de irritabilidade fora do que é comum. & .606 & .137 & .027 & .438 \\
\hline 33. Manifestações de raiva descontrolada. & .596 & .200 & -.083 & .579 \\
\hline 23. Relacionamento conflituoso com as pessoas próximas. & .561 & .085 & -.125 & .470 \\
\hline 16. Resultados escolares bastante inferiores ao esperado. & .557 & -.188 & -.278 & .462 \\
\hline $\begin{array}{l}\text { 35. Envolver-se em atividades de alto risco, quando essas atividades não eram praticadas } \\
\text { com regularidade. }\end{array}$ & .481 & .265 & -.076 & .479 \\
\hline 14. Falar frequentemente sobre atos suicidas. & .025 & .795 & .026 & .631 \\
\hline 26. Ameaçar que vai cometer atos suicidas. & -.017 & .778 & .029 & .575 \\
\hline 22. Aumento do interesse e das conversas sobre o tema do suicídio. & .120 & .708 & .136 & .503 \\
\hline 12. Verbalização de ideias associadas à morte. & -.055 & 639 & -.127 & .462 \\
\hline 28. Dizer que não tem razões para viver. & .082 & .629 & -.104 & .527 \\
\hline 13. Conversas que revelem desinteresse pelo "valor" da vida. & .100 & .519 & -.194 & .475 \\
\hline 4. Desabafos de que a sua vida não faz sentido. & -.091 & .495 & -.357 & .463 \\
\hline 11. Transmitir sentimentos de desapontamento face ao mundo e aos outros. & .254 & .435 & -.098 & .427 \\
\hline 31. Isolamento da sociedade. & .073 & .088 & -.778 & .751 \\
\hline 30. Isolamento da família. & .112 & .016 & -.776 & .727 \\
\hline 2. Isolamento social, afastamento das pessoas com quem convivia habitualmente. & -.033 & .113 & -.774 & .659 \\
\hline 17. Afastamento repentino do grupo de amigos. & .171 & -.034 & -.710 & .647 \\
\hline Valor Próprio (eigenvalues) & 9.94 & 1.99 & 1.17 & \\
\hline Variância Explicada (\%) & 41.28 & 8.66 & 5.10 & \\
\hline Variância Explicada Total (\%) & 55.04 & & & \\
\hline
\end{tabular}

Nota: $N=256$. Os itens encontram-se organizados por fatores e, dentro de cada fator, estão ordenados em função dos pesos fatoriais obtidos no fator primário; $h^{2}=$ Comunalidades 
Quadro 3. Índices de Ajustamento para os Modelos Testados - IRSAS

\begin{tabular}{lccccccccccc}
\hline Modelo & $\chi^{2}$ & $g l$ & $\begin{array}{c}\chi 2 \\
l g l\end{array}$ & $\begin{array}{c}\text { RMSEA } \\
\text { (IC) } \\
95 \%\end{array}$ & $\begin{array}{c}\text { SRMS } \\
\mathrm{R}\end{array}$ & GFI & TLI & CFI & PNFI & ECVI & ACAIC \\
\hline 2F (19 itens) & 384.880 & 147 & 2.62 & $\begin{array}{c}.080 \\
(.070-.089)\end{array}$ & .053 & .864 & .880 & .897 & .726 & 1.847 & 666.323 \\
3F (23 itens) & 435.154 & 215 & 2.02 & $\begin{array}{c}.063 \\
(.055-.072)\end{array}$ & .049 & .871 & .919 & .931 & .742 & 2.185 & 834.410 \\
\hline
\end{tabular}

Nota: $N=256 . \chi^{2}=$ Qui-quadrado; $g l=$ Graus de liberdade; RMSEA = Raiz da média quadrática do erro de aproximação; SRMSR = Raiz da média quadrática residual; GFI = Índice de bondade do ajustamento; TLI = Índice Tucker-Lewis; CFI = Índice de ajustamento comparativo; PNFI = Índice de ajustamento normalizado de parcimónia; ECVI = Índice esperado de validação cruzada. ACAIC - Critério de informação de Akaike. Modelo 2F (19 itens) - Fator 1: 8, 16, 17, 19, 20, 23, 24, 34, 36, 37; Fator 2: 4, 11, 12, 13, 14, 22, 26, 28, 32. Modelo 3F (23 itens) - Fator 1: 8, 16, 23, 24, 27, 29, 33, 34, 35, 36, 37; Fator 2: 4, 11, 12, 13, 14, 22, 26, 28; Fator 3: 2, 17, 30, 31

satisfatórios). De referir que os testes previamente realizados para esta solução garantiram a fatorialidade dos dados, uma vez que o teste de Esfericidade de Bartlett foi significativo $\left(\chi^{2}{ }_{(253)}=\right.$ 2881.609, $p$ <.001) e o KMO foi de .929 (Quadro 2).

Esta solução fatorial explicou $54.45 \%$ da variância total e apresentou 3 fatores com alfas de Cronbach bastante satisfatórios, valores próprios superiores à unidade e percentagem de variância explicada superior a $5 \%$. Os itens têm "pesos fatoriais" superiores a .4 , no respetivo fator primário e inferiores a .3 , no fator secundário, exceto o item 4, que assume um valor ligeiramente superior, mas dada a sua importância teórica optou-se por não o excluir. A matriz de saturação é justificável do ponto de vista teórico. $O$ fator 1 representa Sinais comportamentais $(\alpha=$ $.88)$, o fator 2 Sinais verbais $(\alpha=.86)$ e o fator 3 Sinais de isolamento $(\alpha=.87)$.

De seguida, o IRSAS foi submetido a análises fatoriais confirmatórias, com o intuito de confirmar qual dos modelos (2 ou 3 fatores) apresenta o melhor ajustamento aos dados.

Como se pode observar no Quadro 3, o modelo de 2 fatores com 19 itens obtém o valor .08 no RMSEA e valores inferiores a .9 nos índices GFI, TLI e CFI, o que revela um mau ajustamento deste modelo. $\mathrm{O}$ modelo de 3 fatores com 23 itens, assume um valor inferior a .8 no RMSEA, inferior a .05 no SRMSR e valores de TLI e CFI superiores a .9, que sugerem um bom ajustamento do modelo aos dados. Quando se comparam os dois modelos, o ECVI e o ACAIC são inferiores no modelo de 2 fatores, indicando uma melhor estabilidade do mesmo, no entanto no modelo de 3 fatores o valor do $\mathrm{X}^{2} / \mathrm{gl}$ é menor, sugerindo um ajustamento mais aceitável do mesmo.

Perante os indicadores das análises fatoriais, a opção mais inteligível recai sobre o modelo de 3 fatores com 23 itens, pois apresenta um melhor ajustamento aos dados de acordo com alguns dos índices de ajustamento e permite avaliar três tipologias de sinais de alerta.

Neste sentido, foram explorados os coeficientes padronizados de saturação para os 23 itens do IRSAS e os respetivos indicadores de fiabilidade individual $\left(\mathrm{R}^{2}\right.$, correspondente à fração de variabilidade total do item explicada pelo fator que lhe está associado).

Como pode ser observado no Quadro 4, o valor dos coeficientes de saturação é moderado ou forte (Fornell \& Larcker, 1981). Os indicadores de fiabilidade individual mostraram que o valor mais baixo foi de .315 , no item 29 , e o valor mais elevado foi de .806 , no item 31 .

A análise seguinte consistiu no estudo da correlação entre os fatores através do coeficiente de correlação de Pearson. Os resultados evidenciam correlações moderadas do fator $1 \mathrm{com}$ o fator $2(r=.57 ; p<.001)$ e com o fator $3(r=$ $.67 ; p<.000)$ e também entre o fator 2 e o fator 3 $(r=.63 ; p<.000)$, o que sugere que estes não são totalmente independentes, tal como se pressupôs à partida. 
Quadro 4. Estrutura Fatorial do IRSAS (3F, 23 itens): Coeficiente de Saturação Fatorial (Padronizado) e Indicadores Individuais de Fiabilidade $\left(\mathrm{R}^{2}\right)$

\begin{tabular}{lllll}
\hline Itens & F1 & F2 & F3 & $R^{2}$ \\
\hline 37 & .771 & & & .595 \\
33 & .747 & & & .559 \\
34 & .745 & & & .556 \\
36 & .733 & & & .538 \\
24 & 704 & & & .495 \\
27 & .692 & & & .479 \\
35 & .683 & & & .466 \\
23 & .630 & & & .396 \\
8 & .606 & & & .367 \\
16 & .578 & & & .335 \\
29 & .561 & & & .315 \\
28 & & .695 & & .483 \\
13 & & .690 & & .476 \\
14 & & .677 & & .458 \\
26 & & .646 & & .417 \\
11 & & .634 & & .402 \\
12 & & .619 & & .383 \\
4 & & .612 & & .375 \\
22 & & .584 & & .342 \\
31 & & & .898 & .806 \\
30 & & & .875 & .766 \\
2 & & & .673 & .454 \\
17 & & & .657 & .432 \\
\hline
\end{tabular}

Nota: $N=256 . R^{2}=$ Indicadores individuais de fiabilidade; F1=Fator 1; F2=Fator 2; F3=Fator3 $* p<.05 ; * * p<.01 ; * * * p<.001$.

Tendo em consideração estes resultados, bem como a proposta teórica inicial de uma medida unidimensional, testou-se um modelo hierárquico de segunda ordem, introduzindo um novo fator "IRSAS total". Os resultados demonstram um ajustamento satisfatório deste modelo $\left(\chi^{2}(216)=\right.$ 442.167; $\chi^{2} / \mathrm{gl}=2.047 ;$ RMSEA $=.070 ;$ SRMSR $=.057 ; \mathrm{TLI}=.901 ; \mathrm{CFI}=.915 ; \mathrm{GFI}=.842 ; \mathrm{PNFI}$ $=.725 ; \mathrm{ECVI}=2.664 ;$ ACAIC $=596.214)$, uma vez que todos os índices correspondem aos parâmetros de qualidade definidos, exceto o GFI (Kline, 2011; Marôco, 2010).

A fiabilidade do IRSAS foi, de seguida, avaliada através da consistência interna e da estabilidade temporal, considerando-se nestas análises a amostra total $(N=512)$.
A análise da consistência interna do IRSAS foi realizada para cada um dos fatores, bem como para o total dos itens. Em relação à consistência interna, os resultados mostram que todos os fatores (F1, $\alpha=.90 ; F 2, \alpha=.85 ; F 3, \alpha=.86)$ e o total do IRSAS $(\alpha=.93)$ obtiveram alfas de Cronbach satisfatórios, o que assegura a sua consistência interna. Os coeficientes de correlação item-total corrigidos (ritc) de cada item apresentaram valores adequados (ritc $\geq .54$ ). Também não se registariam alterações significativas nos valores de alfa, caso algum item fosse eliminado.

Para estudar a estabilidade temporal do IRSAS, avaliaram-se os coeficientes de correlação e as diferenças entre os valores médios das duas aplicações, num intervalo de 3 a 4 semanas (Quadro 5).

Os valores médios do primeiro e do segundo momento de aplicação encontraram-se correlacionados de forma positiva e significativa, sendo os coeficientes considerados moderados $(r \geq .53 ; p<.001)$. Não se registaram diferenças significativas nos valores médios entre os dois momentos de aplicação, nem nos três fatores, nem na pontuação total, o que aponta para a constância dos resultados ao longo do tempo.

Desta forma, tendo em conta os resultados ao nível da consistência interna e da estabilidade temporal, poder-se-á afirmar que o IRSAS apresenta bons índices de fiabilidade, pelo que se prosseguiu com o estudo da validade. A validade convergente consiste no grau de coesão dos itens associados a um determinado fator num instrumento psicométrico. Esta validade será tanto melhor quanto maior for a fração da variância dos itens atribuível ao respetivo fator e pode ser avaliada através do índice de Variância extraída média (VEM) (Fornell \& Larcker, 1981). O fator 1 obteve um índice de VEM de .59 , o fator 2 de .54 e o fator 3 de .73 , ou seja, todos registaram valores satisfatórios (>.5).

A validade discriminante foi estudada através do quadrado da correlação $\left(r^{2}\right)$ entre os fatores (variância partilhada), perspetivando-se que este valor seja inferior à variância extraída média de cada fator (Fornell \& Larcker, 1981).

A variância partilhada do fator 1 com o fator 2 $\left(r^{2}=.47\right)$ e com o fator $3\left(r^{2}=.54\right)$ e entre o fator $2 \mathrm{e}$ o fator $3\left(r^{2}=.45\right)$ assumiu valores inferiores aos 
Quadro 5. Estabilidade Temporal do IRSAS (3F, 23 itens)

\begin{tabular}{lcccccc}
\hline & \multicolumn{2}{c}{ Teste } & \multicolumn{2}{c}{ Reteste } & \\
\cline { 2 - 5 } Subescalas do ISAS & $M$ & $D P$ & $M$ & $D P$ & $r$ & $t$ \\
\hline F1 & 3.17 & .640 & 3.21 & .564 & $.59^{* * *}$ & -1.230 \\
F2 & 3.49 & .664 & 3.42 & .610 & $.56^{* * *}$ & 2.035 \\
F3 & 3.50 & .781 & 3.46 & .698 & $.53^{* * *}$ & .920 \\
IRSAS total & 3.34 & .590 & 3.33 & .496 & $.65^{* * *}$ & .479 \\
\hline
\end{tabular}

Nota: $N=300$. $M=$ Média; $D P=$ Desvio-padrão; $r=$ Coeficiente de correlação de Pearson entre os dois momentos de avaliação; $t=$ Teste $t$ de Student para amostras emparelhadas. $* p<.05 ; * * p<.01 ; * * * p<.001$

Quadro 6. Médias, Desvios-Padrão, d de Cohen e teste t de Student em Função do Sexo

\begin{tabular}{lccccccccc}
\hline & $\begin{array}{c}\text { Amostra total } \\
(N=512)\end{array}$ & \multicolumn{2}{c}{$\begin{array}{c}\text { Sexo } \\
\text { masculino } \\
(n=210)\end{array}$} & $\begin{array}{c}\text { Sexo feminino } \\
(n=302)\end{array}$ & & & \\
& $M$ & $D P$ & $M$ & $D P$ & $M$ & $D P$ & $\begin{array}{c}d \text { de } \\
\text { Cohen }\end{array}$ & $t$ & $p$ \\
\hline$F 1$ & 3.20 & .673 & 3.14 & .646 & 3.23 & .690 & -.13 & -1.479 & .140 \\
$F 2$ & 3.50 & .663 & 3.48 & .679 & 3.52 & .651 & -.06 & -.732 & .465 \\
$F 3$ & 3.52 & .785 & 3.43 & .792 & 3.58 & .775 & -.19 & -2.148 & $.032^{*}$ \\
IRSAS total & 3.36 & .601 & 3.31 & .600 & 3.39 & .601 & -.13 & -1.559 & .120 \\
\hline
\end{tabular}

Nota: $N=512 ; M=$ Média; $D P=$ Desvio-padrão; $t=$ Teste $t$ de Student para amostras independentes

da variância extraída média de cada fator, sendo que apenas um valor é igual (.54).

A validade por referência a critério, por sua vez, foi analisada através do coeficiente de correlação de Pearson entre os resultados no IRSAS e nas medidas de resultado referentes à predisposição para a prevenção, nomeadamente a perceção global de sucesso da prevenção do suicídio $(r=.21 ; \quad p<.001)$, a perceção de autoeficácia na prevenção do suicídio dos pares $(r=.28 ; p<.001)$ e a perceção de sucesso na prevenção do suicídio do próprio $(r=.27 ; p<.001)$.

Os coeficientes de correlação foram todos positivos e significativos, embora com valores relativamente baixos. Tal poderá sugerir que um maior reconhecimento dos sinais de alerta está associado a uma maior predisposição para a prevenção.

Por último, procurou-se a existência de diferenças no IRSAS em função do sexo dos participantes através do teste $t$ de Student para amostras independentes (Quadro 6).

Os resultados mostram que não se registaram diferenças significativas entre os sexos nos fatores
1 e 2, nem na pontuação total. Apenas no fator 3, que é relativo aos Sinais de isolamento, se observou que o sexo feminino registou um valor significativamente mais elevado do que o sexo masculino $\quad\left(t_{(510)}=-2.148 ; \quad p<.05\right)$, embora a magnitude da diferença seja reduzida ( $d$ de Cohen=.19) (Marôco, 2011).

\section{Discussão}

O presente estudo teve como principal objetivo desenvolver um inventário de reconhecimento de sinais de alerta para atos suicidas (IRSAS) em adolescentes, cujas características psicométricas fossem satisfatórias.

O desenvolvimento do inventário seguiu os procedimentos recomendados na literatura (Almeida \& Freire, 2008; Gutierrez \& Osman, 2008; Hill \& Hill, 2008; Moreira, 2004; Ribeiro, 1999). Procurou-se elaborar um conjunto de itens o mais representativo possível dos vários sinais de alerta; submeteu-se a versão inicial do instrumento (37 itens) a apreciação por um painel 
de juízes e testou-se numa pequena amostra de adolescentes, o que permitiu garantir a validade de conteúdo do instrumento (Almeida \& Freire, 2008; Hill \& Hill, 2008; Moreira, 2004).

De seguida, aplicou-se o IRSAS a uma amostra total de 512 adolescentes. No que respeita à estatística descritiva e especificamente às medidas de tendência central, pode referir-se que os valores médios, globalmente acima do ponto intermédio da escala de resposta, podem significar que os jovens apresentam um conhecimento mediano sobre os sinais de alerta. Também o facto de todos os sinais serem verdadeiros e de estarem formulados pela positiva pode ter contribuído para que os jovens assinalassem os níveis mais elevados da escala de resposta. Ainda assim, o item com a pontuação mais elevada, o item 25 "Mostrar desinteresse pela vida", registou o valor médio de 3.82 ( $\mathrm{DP}=.853)$ que não é muito elevado, considerando que o máximo é 5. Em relação ao conteúdo deste item, é compreensível que a demonstração de desinteresse pelo valor da vida possa corresponder a um sinal de alerta "quase intuitivo", pois está diretamente relacionado com o suicídio. Quanto ao valor médio, o facto de não ser muito elevado mostra que os jovens não estão completamente convictos de que este pode ser um sinal de alerta para o suicídio, o que remete para a necessidade de mais informação acerca deste assunto. Relativamente ao valor médio mais baixo $(\mathrm{M}=2.89$; $\mathrm{DP}=1.057)$, registado no item 29 "Dificuldade em dormir ou dormir demais, quando isso não é habitual”, pode dever-se à menor valorização que os jovens geralmente fazem dos aspetos mais fisiológicos, como é o caso de dormir, enquanto indicador de uma possível perturbação.

Em relação às medidas de distribuição analisadas, nomeadamente a assimetria e a curtose, os resultados obtidos foram todos inferiores à unidade, $\mathrm{o}$ que sugere que a sensibilidade dos itens na escala não está comprometida (Almeida \& Freire, 2008; Kline, 2011; Marôco, 2011; Moreira, 2004).

A análise dos coeficientes de correlação itemtotal corrigidos demonstra que os itens apresentam uma validade interna adequada. A eliminação de qualquer um deles também não se refletiria numa melhoria significativa do alfa de Cronbach. Estes dados indicam assim que a maioria dos itens apresenta um bom poder discriminativo e o IRSAS apresenta uma consistência interna muito adequada (Almeida \& Freire, 2008; Moreira, 2004; Ribeiro, 1999).

Garantidas estas condições, passou-se à análise fatorial exploratória, testando-se inicialmente os dados no sentido de perceber se coincidiam com o modelo teórico unidimensional concetualizado. Como a versão unifatorial com 37 itens obteve uma variância explicada inferior ao mínimo exigido (50\%) (Marôco, 2011), testaramse soluções fatoriais de 2 e 3 fatores, de acordo com o que era sugerido pelo scree plot e da Simulação de Monte Carlo, indicadores fiáveis do número de fatores a reter (Moreira, 2004; Pestana \& Gageiro, 2009).

As análises fatoriais exploratórias e confirmatórias, em conjunto com os pressupostos concetuais subjacentes à conceção do instrumento, permitiram selecionar o modelo de 3 fatores, com 23 itens, a melhor representação do IRSAS.

O modelo de 3 fatores (23 itens) obteve índices (absolutos e relativos) de ajustamento melhores do que o modelo de 2 fatores, nomeadamente ao nível do CFI, TLI, RMSEA e SRMSR. Quando se comparam os dois modelos com base nos índices ECVI e ACAIC, o modelo de 2 fatores regista valores inferiores; no entanto, não podemos adotar este modelo, uma vez que os restantes índices sugerem um pior ajustamento aos dados. O valor $\mathrm{X} 2 / \mathrm{gl}$ no modelo 3 também sugere um melhor ajustamento do mesmo. Perante estes resultados, globalmente o ajustamento do modelo de 3 fatores é considerado bom (Marôco, 2010). Além disso, em termos de interpretação teórica, o modelo de 3 fatores, Faculta informação sobre três tipos de sinais de alerta: Sinais comportamentais (fator 1), Sinais verbais (fator 2) e Sinais de isolamento (fator 3). De referir que alguns autores têm também discriminado os sinais de alerta em dois grupos principais: Sinais comportamentais e Sinais verbais (e.g., Inoue et al., 2007). Os Sinais de isolamento, por sua vez, embora se manifestem de forma comportamental assumem uma especificidade distinta de outros sinais comportamentais, o que justifica a sua autonomização num fator. Além disso, o 
isolamento é uma das principais características do risco suicida (e.g., Berman et al., 2006; Capuzzi, 2009).

Assim, deu-se continuidade ao estudo das propriedades psicométricas do modelo de 3 fatores (23 itens). No parâmetro dos coeficientes padronizados de saturação fatorial, os resultados foram maioritariamente considerados moderados ou fortes (Fornell \& Larcker, 1981), tal como desejável neste tipo de instrumentos.

As correlações entre os fatores evidenciam que existe associação moderada entre os mesmos, o que teoricamente é justificável devido à própria natureza dos sinais de alerta sendo que estes sinais podem estar relacionados entre si e não se excluem mutuamente (AAS, 2013; Berman et al., 2006; Rudd, Berman, et al., 2006), o que explica a relação entre Sinais comportamentais (fator 1), Sinais verbais (fator 2) e Sinais de isolamento (fator 3). Considera-se, assim, que a diferenciação dos sinais de alerta nestes fatores encontra interpretação teórica plausível, sendo igualmente aceitável a associação registada entre os mesmos, pelas razões já explicadas.

Foi ainda testado um modelo hierárquico com um fator de segunda ordem - IRSAS total - que obteve um bom ajustamento aos dados. Este resultado, por um lado, suporta a estrutura correlacional encontrada e, por outro lado, vai ao encontro da perspetiva unidimensional que esteve na base do desenvolvimento do IRSAS. A análise do alfa de Cronbach dos fatores revelou uma boa consistência interna. No parâmetro da estabilidade temporal, os resultados de ambas as aplicações estão significativamente correlacionados não existindo diferenças significativas do ponto de vista estatístico entre as mesmas. Neste sentido, a fiabilidade do IRSAS, medida pelos critérios de consistência interna e estabilidade temporal (Almeida \& Freire, 2008; Moreira, 2004; Pestana \& Gageiro, 2009; Ribeiro, 1999), está garantida.

Uma vez que a avaliação da fiabilidade do IRSAS foi bastante adequada, prosseguiu-se com o estudo das validades.

No que respeita à validade convergente, os índices de VEM são adequados em todos os fatores, garantido o critério mínimo de exigência (>.5) (Fornell \& Larcker, 1981). Pode, deste modo, afirmar-se que existe coesão dos itens nos respetivos fatores.

No parâmetro relativo à validade discriminante, a variância partilhada foi inferior à VEM, sendo que apenas um valor é similar em ambas as análises, porém não coloca dúvidas quanto à diferenciação dos fatores.

A relação entre o IRSAS e a predisposição para a prevenção, como era expetável, é positiva e significativa, ou seja, quanto maior o reconhecimento dos sinais de alerta para os atos suicidas, maior a predisposição para agir de forma preventiva (Tsai et al., 2010).

Todavia, o grau de associação encontrado entre estas duas variáveis é considerado baixo ( $\mathrm{r}$ .2), o que suscita duas reflexões. Por um lado, o valor baixo da correlação pode dever-se ao facto de terem sido utilizados itens isolados como critérios, por outro lado, também pode significar a necessidade de uma intervenção psicoeducacional no sentido de fortalecer a associação entre as mesmas, isto é, se promovermos a capacidade de reconhecimento dos sinais de alerta provavelmente aumentamos a perceção de eficácia do jovem para prevenir um caso de suicídio. Como a literatura tem evidenciado, o reconhecimento dos sinais de alerta pode ser determinante na prevenção de um ato suicida (Berman et al., 2006; Capuzzi, 2009; Tsai et al., 2010).

Numa última análise foram comparados os resultados médios no ISAS, em função do sexo e registaram-se diferenças significativas no fator 3, Sinais de isolamento. O facto de terem sido as raparigas a pontuar mais elevado no reconhecimento deste tipo de sinais de alerta pode dever-se às próprias características do sexo feminino na adolescência. Normalmente as raparigas quando vivenciam situações de maior instabilidade emocional ou humor negativo tendem a isolar-se e a adotar comportamentos internalizantes, enquanto os rapazes têm normalmente mais comportamentos externalizantes (Sprinthall \& Collins, 2008). Assim, é justificável que as raparigas estejam mais sensibilizadas para identificar como sinais de alerta um tipo de comportamento que experienciam com mais frequência. 
Após a análise das propriedades psicométricas do IRSAS, pode sintetizar-se o seguinte: todos os índices de ajustamento aos dados são satisfatórios, quando avaliados ao nível da análise fatorial confirmatória de primeira e de segunda ordem, exceto o GFI; os coeficientes padronizados de saturação fatorial são adequados; a consistência interna dos fatores é bastante satisfatória, bem como a estabilidade temporal dos resultados entre ambas as aplicações; os resultados correlacionamse da forma perspetivada teoricamente com a medida de resultado e ao nível das diferenças em função do sexo, registam-se diferenças significativas no fator 3, Sinais de isolamento.

De uma forma global, o IRSAS permite avaliar o reconhecimento de um conjunto de sinais de alerta e apresenta características psicométricas consideradas satisfatórias nos diversos parâmetros estudados. Pode, por isso, considerar-se que o IRSAS cumpre os propósitos que pautaram a sua construção e desenvolvimento.

O desenvolvimento de um instrumento é sempre um percurso longo que pode ser melhorado em estudos futuros, colmatando as limitações do presente. Como principais limitações pode referir-se a falta de uma metodologia que efetivamente garanta a validade do instrumento e o facto de se tratar de um teste de conhecimentos, cujo estudo da dificuldade dos itens podia ter sido mais aprofundado. Podem ainda apontar-se outras limitações, como o facto de todos os itens se referirem a sinais de alerta, não havendo itens distractores, o que pode ter facilitado o reconhecimento dos mesmos; o facto de se tratar da primeira aplicação do IRSAS não permite a comparação dos resultados em outros estudos; a inexistência de outros instrumentos com os mesmos objetivos validados em Portugal também não permite $\mathrm{o}$ estudo da validade concorrente; a utilização de itens isolados para avaliar a validade por referência a critério; a eliminação de uma parte dos itens levou à perda de uma parte dos sinais de alerta, embora tivesse sido uma premissa de partida elaborar itens repetidos para poderem ser selecionados os melhores, do ponto de vista psicométrico.

Como sugestões para o futuro, o IRSAS poderá ser estudado numa amostra mais representativa da população portuguesa adolescente, bem como em outros setores etários da população. No âmbito de ações de prevenção primária, poderá ser utilizado especificamente em estudos dirigidos à comunidade em geral ou a familiares e pessoas próximas de indivíduos em risco de suicídio, como instrumento de partida para o rastreio de conhecimentos sobre sinais de alerta e para avaliar a evolução do grau de conhecimentos antes e após ações de prevenção.

A literatura tem documentado que o reconhecimento dos sinais de alerta é um dos aspetos essenciais na prevenção do suicídio (Berman et al., 2006; Miller, 2011; Shaffer \& Gould, 2000; Waldvogel et al., 2008). Uma vez que o IRSAS mostrou ter propriedades psicométricas satisfatórias, poderá ser potencialmente útil para analisar o grau de reconhecimento dos sinais de alerta.

\section{Referências}

AAS (American Association of Suicidology) (2013). IS PATH WARM? Retrieved from http://www.suicidology.org/web/guest/home.

Almeida, L. S., \& Freire, T. (2008). Metodologia da investigação em psicologia e educação. Braga: Psiquilíbrios.

Arenas-Landgrave, P., Maqueo, E. L. G., \& Forns, M. (2012). Differential personality indicators in the face of suicidal risk in adolescents. Revista Iberoamericana de Diagnostico y Evaluación - e Avaliação Psicológica, 33(1), 51-74.

Aseltine, R. H., \& DeMartino, R. (2004). An outcome evaluation of the SOS suicide prevention program. American Journal of Public Health, 94(3), 446-451. doi: 10.2105/AJPH.94.3.446.

Aseltine, R. H., James, A., Schilling, E. A., \& Glanovsky, J. (2007). Evaluating the SOS suicide prevention program: A replication and extension. Bmc Public Health, 7(161). doi:10.1186/1471-2458-7-161.

Barnes, L. S., Ikeda, R. M., \& Kresnow, M. J. (2001). Help-seeking behavior prior to nearly lethal suicide attempts. Suicide and LifeThreatening Behavior, 32(1), 68-75. doi:10.1521/suli.32.1.5.68.24217. 
Berman, A., Jobes, D., \& Silverman, M. (2006). Adolescent suicide: Assessment and intervention. Washington: American Psychological Association.

Bryan, C. J., Steiner-Pappalardo, N., \& Rudd, M. D. (2009). Exposure to a mnemonic interferes with recall of suicide warning signs in a community-based suicide prevention program. Suicide and Life-Threatening Behavior, 39(2), 194-203.

Capuzzi, D. (2009). Suicide prevention in the schools. Guidelines for middle and high school settings. Alexandria, VA, USA: American Counseling Association.

Carvalho, R. G., \& Novo, R. F. (2014). Personality dimensions and risk behaviours in adolescence: A study with the Portuguese version of the MMPI-A. Revista Iberoamericana de Diagnostico y Evaluación - e Avaliação Psicológica, 1(37), 203-222.

DGS (Direção-Geral de Saúde) (2013). Plano Nacional de Prevenção do Suicídio (2013/2017). Retirado de http://www.portal dasaude.pt/NR/rdonlyres/BCA196AB-74F4472B-B21E-6386D4C7A9CB/0/i018789.pdf 2013 .

Fornell, C., \& Larcker, D. F. (1981). Evaluating structural equation modeling with unobserved variables and measurement error. Journal of Marketing Research, 18(1), 39-50.

Gould, M. S., Greenberg, T., Velting, D. M., \& Shaffer, D. (2003). Youth suicide risk and preventive interventions: A review of the past 10 years. Journal of the American Academy of Child and Adolescent Psychiatry, 42(4), 386405. doi:10.1097/01.CHI.0000046821.95464.CF.

Gould, M. S., Marrocco, F. A., Kleinman, M., Thomas, J. G., Mostkoff, K., Cote, J., \& Davies, M. (2005). Evaluating iatrogenic risk of youth suicide screening programs - A randomized controlled trial. Jama-Journal of the American Medical Association, 293(13), 1635-1643. doi:10.1001/jama.293.13.1635.

Gutierrez, P. M., \& Osman, A. (2008). Adolescent suicide: An integrated approach to the assessment of risk and protective factors. Illinois: Northern Illinois University Press.
Hawton, K. (2010). Restriction of access to methods of suicide as a means of suicide prevention. In K. Hawton (Ed.), Prevention and treatment of suicidal behaviour. From science to practice. (pp. 279-292). New York: Oxford University Press.

Hill, M. M., \& Hill, A. (2008). Investigação por questionário. Lisboa: Edições Sílabo.

Hjelmeland, H., \& Knizek, B. L. (2004). The general public's views on suicide and suicide prevention, and their perception of participating in a study on attitudes towards suicide. Archives of Suicide Research, 8(4), 345-359. doi: 10.1080/13811110490476725.

Inoue, K., Tanii, H., Fukunaga, T., Abe, S., Nishimura, Y., Kaiya, H., . . . Okazaki, Y. (2007). Analysis of pre-suicide signs: Implications for suicide prevention. West Indian Medical Journal, 56(3), 312-312.

Joiner, T. (2010). Myths about suicide. London: Harvard University Press.

Juhnke, G., Granello, P., Lebrón-Striker, M., \& Lebrón-Striker. (2007). IS PATH WARM? A suicide assessment mnemonic for counselors. Professional Counseling Digest.

Kalafat, J., \& Elias, M. (1994). An evaluation of a school-based suicide awareness intervention. Suicide and Life-Threatening Behavior, 24(3), 224-233.

King, K. A. (1999). Fifteen prevalent myths concerning adolescent suicide Commentaries. Journal of School Health, 69(4), 159-161.

Kline, R. B. (2011). Principles and practice of structural equation modeling. New York: The Guilford Press.

Marôco, J. (2010). Análise de equações estruturais. Fundamentos teóricos, software \& aplicações. Pêro Pinheiro: ReportNumber.

Marôco, J. (2011). Análise estatística com o SPSS Statistics. Pero Pinheiro: Report Number.

Miller, D. N. (2011). Child and adolescent suicidal behavior. School-based prevention, assessment, and intervention. New York: The Guildford Press.

Moreira, J. M. (2004). Questionários: Teoria $e$ prática. Coimbra: Livraria Almedina.

OMS (Organização Mundial de Saúde) (2015). Suicide prevention (SUPRE). Retrieved from 
http://www.who.int/mental_health/prevention/ suicide/suicideprevent/en/

Owens, C., Booth, N., Briscoe, M., Lawrence, C., \& Lloyd, K. (2003). Suicide outside the care of mental health services: A case-controlled psychological autopsy study. Crisis: The Journal of Crisis Intervention and Suicide Prevention, 24(3), 113-121. doi:10.1027//022 7-5910.24.3.113.

Pestana, M. H., \& Gageiro, J. N. (2009). Análise de dados para ciências sociais: A complementaridade do SPSS (5. ed.). Lisboa: Edições Sílabo.

Ribeiro, J. L. P. (1999). Investigação e avaliação em psicologia e saúde. Lisboa: Climepsi editores.

Rudd, M. D., Berman, A. L., Joiner, T. E., Nock, M. K., Silverman, M. M., Mandrusiak, M., . . . Witte, T. (2006). Warning signs for suicide: Theory, research, and clinical applications. Suicide and Life-Threatening Behavior, 36(3), 255-262. doi:10.1521/suli.2006.36.3.255.

Rudd, M. D., Mandrusiak, M., Joiner, T. E., Berman, A. L., Van Orden, K. A., \& Hollar, D. (2006). The emotional impact and ease of recall of warning signs for suicide: A controlled study. Suicide and LifeThreatening Behavior, 36(3), 288-295. doi:10.1521/suli.2006.36.3.288.

Shaffer, D., \& Gould, M. (2000). Suicide prevention in schools. In K. Hawton \& K. van Heeringen (Eds.), The international handbook of suicide and attempted suicide. Chichester, UK: John Wiley \& Sons, Ltd.

Sprinthall, N. A., \& Collins, W. A. (2008). Psicologia do adolescente. Lisboa: Fundação Calouste Gulbenkian.

Tsai, W. P., Lin, L. Y., Chang, W. L., Chang, H. C., \& Chou, M. C. (2010). The effects of the suicide awareness program in enhancing community volunteers' awareness of suicide warning signs. Archives of Psychiatric Nursing, 24(1), 63-68. doi:10.1016/j.apnu.200 9.01.004.

Van Orden, K. A., Joiner, T. E., Hollar, D., Rudd, M. D., Mandrusiak, M., \& Silverman, M. M. (2006). A test of the effectiveness of a list of suicide warning signs for the public. Suicide and Life-Threatening Behavior, 36(3), 272287. doi:10.1521/suli.2006.36.3.272.

Waldvogel, J. L., Rueter, M., \& Oberg, C. N. (2008). Adolescent suicide: Risk factors and prevention strategies. Curr Probl Pediatr Adolesc Health Care, 38(4), 110-125. doi:10.1016/j.cppeds.2008.01.003.

Walker, R. L., Ashby, J., Hoskins, O. D., \& Greene, F. N. (2009). Peer-support suicide prevention in a non-metropolitan US community. Adolescence, 44(174), 335-346. 